\title{
A Generalized Fuzzy Entropy-Based Image Segmentation Method
}

\author{
Jiulun Fan $^{1}$ Feng Zhao ${ }^{2}$ \\ ${ }^{1}$ Department of Information and Control, Xi'an Institute of Post and Telecommunications, Xi'an 710061, P.R. China \\ ${ }^{2}$ Institute of Intelligent Information Processing, Xidian University, Xi'an 710071, P.R. China
}

\begin{abstract}
Image segmentation using fuzzy entropy is an important and common segmentation method. The threshold of fuzzy entropy is mostly selected at the gray value with fuzzy membership degree 0.5 . It is a limitation in some cases. In order to solve this problem, we present a new definition of generalized fuzzy entropy and apply it to image segmentation. Compared with the traditional fuzzy entropy-based image segmentation method, the proposed method segments an image using the threshold with membership degree $\mathrm{m}(0<\mathrm{m}<1)$, and increases the opportunity of choosing appropriate thresholds. Experiments show that our method can obtain better segmentation result than the traditional fuzzy entropy method.
\end{abstract}

Keywords: Image segmentation, Fuzzy entropy, Generalized fuzzy entropy, Fuzzy complement operator

\section{Introduction}

Fuzzy entropy describes the fuzziness of a fuzzy set. It is a basic concept in fuzzy set theory and widely used in image processing. For example, fuzzy entropybased segmentation method is a useful kind of segmentation method [1-3]. The first definition of fuzzy entropy on finite universal set was introduced by De Luca and Termini [4]. They used the basic union, intersection and complement operators $c(x)=1-x \quad(x \in[0,1])$ defined by Zadeh [5]. A notable feature of this complement operator is that its fixed point locates at $x=0.5$, this meaning that the fuzzy set $A=[0.5]$ (i.e., $\mu_{A}(x) \equiv 0.5$ for all $x$ in the universal set) has the most fuzziness.

Fuzzy set $A=[0.5]$ possessing the most fuzziness is a limitation in real applications. If we use a measure of fuzziness to assess the amount of blur in an image, as pointed out by $\mathrm{Pal}$ [6], the use of the fixed peek at 0.5 may lead to inaccurate estimates. Zenzo et al. [7] changed the conditions of fuzzy entropy, replacing the condition that fuzzy set $A=[0.5]$ has the most fuzziness with fuzzy set $A=[m](m \in(0,1))$ has the most fuzziness. Using a mapping, they constructed a generalized fuzzy entropy formula based on fuzzy entropy formula, and applied it to image segmentation and denoising. Unfortunately, the definition of Zenzo's generalized fuzzy entropy did not involve the complement operator of a fuzzy set, that is, the fourth condition in the definition of fuzzy entropy was abandoned. In this paper we presents a complete definition of generalized fuzzy entropy which is entirely corresponding to the conditions of traditional fuzzy entropy, and give an image segmentation method based on it. The method segments an image using the threshold with membership degree $\mathrm{m}$ $(0<\mathrm{m}<1)$.

The paper is organized as follows. In Section 2, we briefly state and review the definition of fuzzy entropy. In Section 3, a complete definition of generalized fuzzy entropy is proposed. Section 4 presents an image segmentation method based on the generalized fuzzy entropy. The contrastive segmentation performance of the generalized fuzzy entropy with that of the fuzzy entropy is provided in Section 5. Finally, conclusions and future works are discussed in Section 6.

\section{Fuzzy entropy}

Throughout this paper, $\mathbf{F}(X)$ expresses the set of all fuzzy sets on universal set $X$. $\mathbf{P}(X)$ expresses the set of all crisp sets on universal set $X . \mu_{A}(x)$ is the membership function of $A \in \mathbf{F}(X)$. [a] is the fuzzy set of $X$ for which $\mu_{[a]}(x)=a, \forall x \in X(a \in[0,1])$. For fuzzy set $A$, we use $A^{c}$ to express the complement of $A$ where $c(x)=1-x(x \in[0,1])$. For two fuzzy sets $A$ and $B, A \cup B$ the union of $A$ and $B$ is defined as $\mu_{A \cup B}(x)=\max \left(\mu_{A}(x), \mu_{B}(x)\right)$, and $A \cap B$ the intersection of $A$ and $B$ is defined as $\mu_{A \cap B}(x)=\min \left(\mu_{A}(x), \mu_{B}(x)\right)$. A fuzzy set $A^{*}$ is called a sharpening of $A$. If $\mu_{A}(x) \geq 0.5$, then $\mu_{A^{*}}(x) \geq \mu_{A}(x)$; if $\mu_{A}(x) \leq 0.5$, then $\mu_{A^{*}}(x) \leq \mu_{A}(x)$.

In order to measure the fuzziness of a fuzzy set on finite universal set $X=\left\{x_{1}, x_{2}, \ldots x_{n}\right\}$, De Luca and 
Termini [4] introduced the definition of fuzzy entropy. Fuzzy entropy is a function $e: \quad \mathbf{F}(X)$ $\rightarrow R^{+}\left(R^{+}=[0,+\infty)\right)$, which satisfies the following four conditions:

(EP1) $e(A)=0$ iff $A \in \mathbf{P}(X)$;

(EP2) $e(A)$ attains its maximum iff $A=[0.5]$;

(EP3) If $A^{*}$ is a sharpening of $A$, then $e\left(A^{*}\right) \leq e(A)$;

(EP4) $e\left(A^{c}\right)=e(A)$.

The above four conditions are basic and reasonable for the measure of fuzziness of a fuzzy set. (EP4) requires the fuzzy set $A$ and $A^{c}$ possess the same fuzziness.

\section{Generalized fuzzy entropy}

The complement operator $c(x)=1-x$ has a unique fixed point $x=0.5$.This complement operator is widely used in fuzzy set theory and applications. Many researchers have proposed general fuzzy complement operators [8-11]. For these fuzzy complement operators, the unique fixed point can be any point in the internal $(0,1)$. The general fuzzy complement operator $c:[0,1] \rightarrow[0,1]$ must satisfy

(C1) $c(0)=1$ and $c(1)=0$;

(C2) For all $a, b \in[0,1]$, if $a \leq b$ then $c(a) \geq c(b)$;

(C3) $c$ is a continuous function;

(C4) For every point $x \in[0,1], c(c(x))=x$.

Yager [10] gave a fuzzy complement function:

$$
c_{w}(x)=\left(1-x^{w}\right)^{1 / w}
$$

where $w \in(0,+\infty)$. When $w=1$, Yager's complement turns to the fuzzy complement function $c(x)=1-x$. The unique fixed point of $c_{w}(x)$ is $m=\frac{1}{\sqrt[w]{2}}$.

Zenzo et al. [7] had given a definition of generalized fuzzy entropy, but their definition omitted the fourth condition (EP4) making their definition too loose. In order to give a complete definition of generalized fuzzy entropy which is entirely corresponding to the conditions of fuzzy entropy, we propose a new definition of generalized fuzzy entropy on a finite universal set. For $m \in(0,1)$ and a complement operator $c_{m}$ with the fixed point $m$, the generalized fuzzy entropy is a function $e_{m}$ : $\mathbf{F}(X) \rightarrow R^{+}$, which satisfies the following four conditions:

(GEP1) $e_{m}(A)=0$ iff $A \in \mathbf{P}(X)$;

(GEP2) $e_{m}(A)$ attains its maximum iff $A=[m]$;
(GEP3) If $A^{*}$ is a sharpening of $A$, then $e_{m}\left(A^{*}\right) \leq e_{m}(A)$. Here $A^{*}$ satisfies that $\mu_{A^{*}}(x) \geq \mu_{A}(x)$ when $\mu_{A}(x) \geq m ; \mu_{A^{*}}(x) \leq \mu_{A}(x)$ when $\mu_{A}(x) \leq m$;

(GEP4) $e\left(A^{c_{m}}\right)=e(A)$.

With $m=0.5$ and fuzzy complement function $c(x)=1-x$, the generalized fuzzy entropy comes to the fuzzy entropy.

For the fuzzy set $A$ on a finite universal set $X=\left\{x_{1}, x_{2}, \ldots x_{n}\right\}$, some generalized fuzzy entropy formulas are given below

$$
\begin{aligned}
e_{m}^{1}(A) & =\frac{M\left(A \cap A^{c_{m}}\right)}{M\left(A \bigcup A^{c_{m}}\right)} \\
e_{m}^{2}(A) & =\sum_{i=1}^{n} \frac{\min \left(\mu_{A}\left(x_{i}\right), \mu_{A^{c_{m}}}\left(x_{i}\right)\right)}{\max \left(\mu_{A}\left(x_{i}\right), \mu_{A^{c_{m}}}\left(x_{i}\right)\right)} \\
e_{m}^{3}(A) & =M\left(A \cap A^{c_{m}}\right) \\
e_{m}^{4}(A) & =1-\frac{M\left(A \bigcup A^{c_{m}}\right)-M\left(A \cap A^{c_{m}}\right)}{n}
\end{aligned}
$$

where $M(A)=\sum_{i=1}^{n} \mu_{A}\left(x_{i}\right)$.

\section{Generalized fuzzy entropy-based image segmentation method}

Fuzzy entropy-based thresholding method has become one of the frequently used segmentation technique during these years. As described in Section 3, fuzzy entropy is the special case of the generalized fuzzy entropy, so it is natural to apply the generalized fuzzy entropy to image segmentation problems. We sample the parameter $m$ in the internal $(0,1)$ with a step of 0.1 ,then for each image, nine segmentation results can be obtained. When $m=0.5$, the result is the very one of fuzzy entropy-based method. This means that the generalized fuzzy entropy-based method can obtain performance not worse than that of the fuzzy entropy, because it involves the result of the fuzzy entropy. Among the nine segmentation results, we then select the one which has the best visual effect as the final result of the generalized fuzzy entropy.

Let $Q$ denote an image of size $M \times N$ with $L$ levels, and $q_{x y}$ is the gray level of $(x, y)$ pixel in $Q$. Let $\mu_{Q}\left(q_{x y}\right)$ denote the membership value which represents the degree of brightness of $(x, y)$ pixel in $Q$. In the notation of fuzzy set, the image set $Q$ can be written as $Q=\left\{\left(q_{x y}, \mu_{Q}\left(q_{x y}\right)\right\}\right.$, where $0 \leq \mu_{Q}\left(q_{x y}\right) \leq 1$.

In this paper, we use the $S$-function to represent the brightness of gray level. It is defined as [12] 


$$
S(x ; a, b, d)=\left\{\begin{array}{cc}
0, & x \leq a \\
\frac{(x-a)^{2}}{(b-a)(d-a)}, & a \leq x \leq b \\
1-\frac{(x-d)^{2}}{(d-b)(d-a)}, & b \leq x \leq d \\
1, & x \geq d
\end{array}\right.
$$

where $x$ is the gray level in $Q$, and $a, b, d$ are the parameters determining the shape of S-function. Parameter $b$ can be any point between $a$ and $d$.

Fuzzy entropy-based segmentation method always regard the maximum fuzzy entropy as the threshold selecting principle. The shape of S-function is determined by parameter $a, b$ and $d$. Therefore, the threshold selecting problem becomes to find a combination of the parameters such that the corresponding event has the maximum fuzzy entropy $[2,13]$. After obtaining the best combination of the parameters $\left(a^{*}, b^{*}, d^{*}\right)$, the gray level with membership value 0.5 is selected as the threshold. We use $G=\{g(x, y)\}$ to denote the segmented image, then the optimal threshold value is selected at

$$
\begin{gathered}
\left(a^{*}, b^{*}, d^{*}\right)=\operatorname{Arg} \max _{0 \leq a<b<d \leq L-1} e(a, b, d) \\
T^{*}=t, \text { iff } \mu_{Q}(t)=0.5 \\
g(x, y)= \begin{cases}0 & q(x, y) \leq T^{*} \\
1 & q(x, y)>T^{*}\end{cases}
\end{gathered}
$$

As described in Introduction, selecting the threshold value with membership degree 0.5 is a limitation in real applications. Next we give the generalized fuzzy entropy-based threshold method. The optimal threshold value is selected at

$$
\begin{gathered}
\left(a^{*}, b^{*}, d^{*}\right)=\operatorname{Arg} \max _{0 \leq a<b<d \leq L-1} e_{m}(a, b, d) \\
T^{*}=t, \text { iff } \mu_{Q}(t)=m \\
g(x, y)= \begin{cases}0 & q(x, y) \leq T^{*} \\
1 & q(x, y)>T^{*}\end{cases}
\end{gathered}
$$

When we use Yager's complement function to substitute the complement operator $c(x)=1-x$ in (2) (5), parameters $w$ is included in these formulas. Because $m$ is the fixed point, thus the relation between $m$ and $w$ is:

$$
w=-\frac{1}{\log _{2} m}
$$

Formula (13) maps $m \in(0,1)$ onto $w \in(0,+\infty)$. Using formula (13), Yager's complement can be expressed as

$$
c_{m}(x)=\left(1-x^{-\frac{1}{\log _{2} m}}\right)^{-\log _{2} m}
$$

By formula (14), the generalized entropy formulas (2) (5) contained the parameter $m(m \in(0,1))$.

We sample the parameter $m$ in the internal $(0,1)$ with a step of 0.1 . So for each image, nine segmentation results can be obtained. Among the nine segmentation results, we select the one which has the best visual effect as the final result of the generalized fuzzy entropy.

\section{Experimental results}

In this section, we compare the segmentation performance of the generalized fuzzy entropy with that of the traditional fuzzy entropy-based method. The generalized fuzzy entropy formulas are formula (2) (5). The complement function is Yager's complement. The experimental images are Rice, Circuit board and Car number. Table 1 3 list the thresholds under the four generalized fuzzy entropy formulas, when $m$ equals $0.1,0.2, \cdots, 0.9$, respectively. When $m=0.5$, the threshold is the threshold of traditional fuzzy entropybased method. In Table 1 3, under each generalized fuzzy entropy formula, every overstriking data is the final threshold selected from the nine thresholds with the best visual effect.

\begin{tabular}{|c|c|c|c|c|}
\hline$m$ & $e_{m}^{1}$ & $e_{m}^{2}$ & $e_{m}^{3}$ & $e_{m}^{4}$ \\
\hline 0.1 & 92 & 102 & 100 & 92 \\
\hline 0.2 & 108 & 105 & 105 & 113 \\
\hline 0.3 & 105 & 105 & 105 & 105 \\
\hline 0.4 & 101 & 102 & 101 & 101 \\
\hline 0.5 & 88 & 100 & 88 & 88 \\
\hline 0.6 & 101 & 101 & 101 & 101 \\
\hline 0.7 & 114 & $\mathbf{1 1 0}$ & 114 & 114 \\
\hline 0.8 & $\mathbf{1 3 1}$ & 107 & $\mathbf{1 3 1}$ & $\mathbf{1 3 1}$ \\
\hline 0.9 & 152 & 152 & $` 152$ & 152 \\
\hline
\end{tabular}

Table1.The threshold of Rice image

\begin{tabular}{|c|l|c|c|c|}
\hline$m$ & $e_{m}^{1}$ & $e_{m}^{2}$ & $e_{m}^{3}$ & $e_{m}^{4}$ \\
\hline 0.1 & 71 & 199 & 71 & 71 \\
\hline 0.2 & 100 & 199 & 100 & 100 \\
\hline 0.3 & $\mathbf{1 2 2}$ & $\mathbf{1 2 2}$ & $\mathbf{1 2 2}$ & $\mathbf{1 2 2}$ \\
\hline 0.4 & 141 & 141 & 141 & 141 \\
\hline 0.5 & 158 & 158 & 158 & 158 \\
\hline 0.6 & 173 & 173 & 173 & 173 \\
\hline 0.7 & 186 & 186 & 186 & 186 \\
\hline 0.8 & 124 & 124 & 124 & 124 \\
\hline 0.9 & 153 & 153 & 153 & 153 \\
\hline
\end{tabular}

Table2. The threshold of Circuit board image

\begin{tabular}{|c|c|c|c|c|}
\hline$m$ & $e_{m}^{1}$ & $e_{m}^{2}$ & $e_{m}^{3}$ & $e_{m}^{4}$ \\
\hline 0.1 & 108 & 119 & 116 & 83 \\
\hline 0.2 & 115 & 116 & 115 & 115 \\
\hline 0.3 & 128 & 127 & 128 & 129 \\
\hline 0.4 & 128 & 128 & 128 & 128 \\
\hline 0.5 & 116 & 119 & 116 & 116 \\
\hline 0.6 & 101 & 108 & 102 & 102 \\
\hline 0.7 & 117 & 117 & 117 & 117 \\
\hline 0.8 & 142 & 142 & 142 & 142 \\
\hline 0.9 & $\mathbf{1 7 5}$ & $\mathbf{1 7 5}$ & $\mathbf{1 7 5}$ & $\mathbf{1 7 5}$ \\
\hline
\end{tabular}

Table3. The threshold of Car number image 


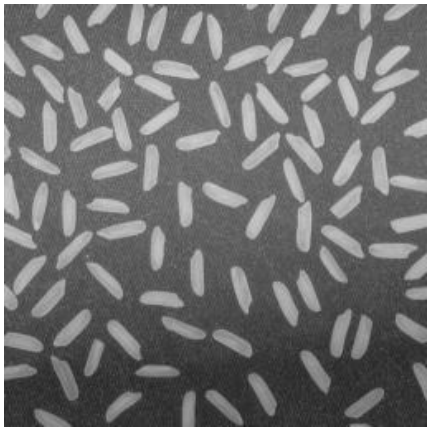

(a)

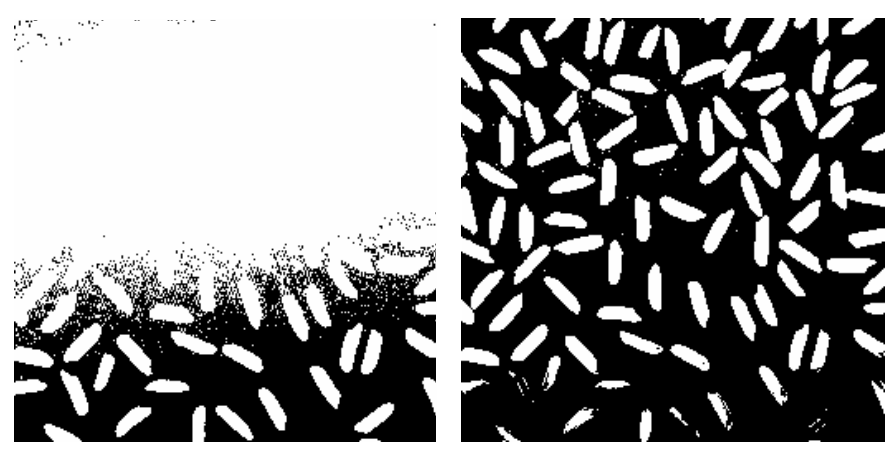

(b)

(c)

Figure.1 The result of Rice image: (a) original image ; (b) segmentation by fuzzy entropy ; (c) segmentation by generalized fuzzy entropy.

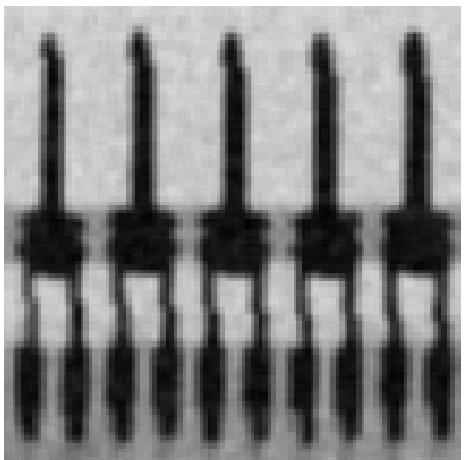

(a)

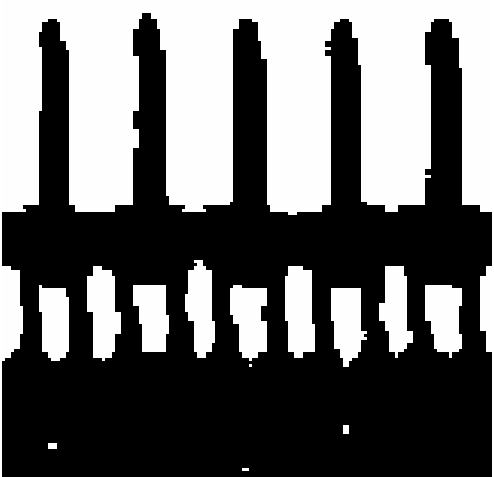

(b)

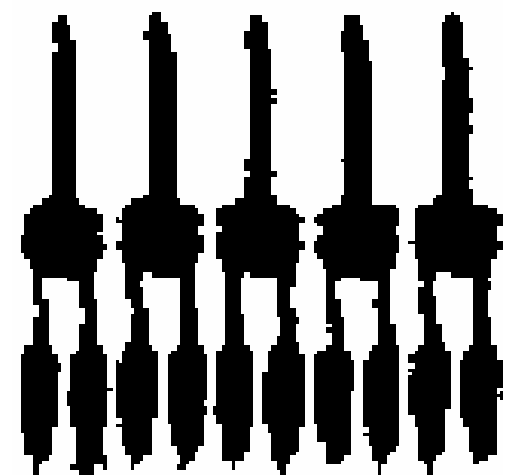

(c)

Figure.2 The result of Circuit board image: (a) original image ; (b) segmentation by fuzzy entropy ; (c) segmentation by generalized fuzzy entropy.
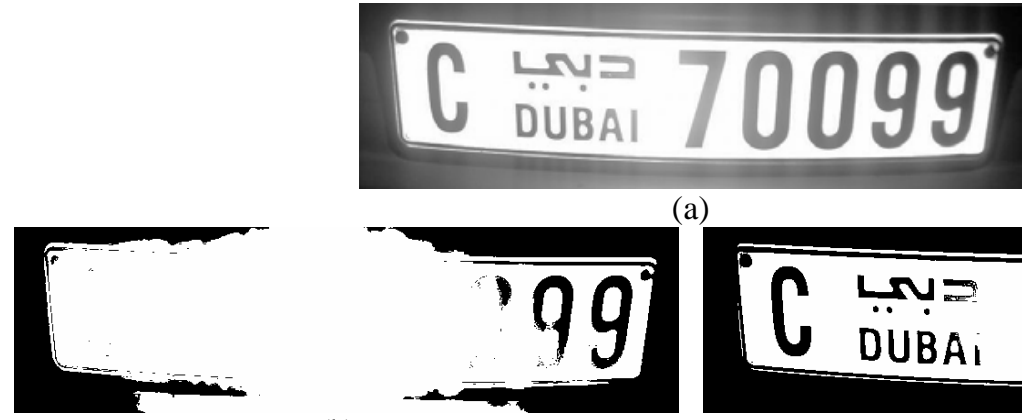

(a)

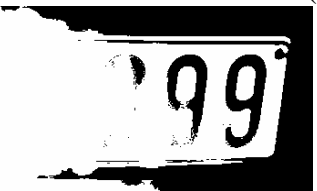

(b)

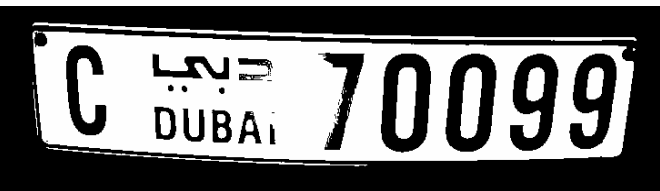

(c)

Figure.3 The result of Car number board image: (a) original image ; (b) segmentation by fuzzy entropy ; (c) segmentation by generalized fuzzy entropy.

From Table. $1 \sim 3$, we can see that for each image, the thresholds of the four generalized fuzzy entropy formulas are almost the same under the same $m$ value. So we only present the segmentation results of the formula $e_{m}^{1}$, listed in Fig. $1 \sim 3$.

For Rice image, under the generalized fuzzy entropy formula $e_{m}^{1}$, the threshold of fuzzy entropy is 88, and the final threshold of the generalized fuzzy entropy is located at $m=0.8(\mathrm{~T}=131)$ (see Table 1 ). Fig.1.(b) and (c) list the two segmentation results. We can see that the generalized fuzzy entropy obtains more satisfactory result than the fuzzy entropy. Similar analysis of other images is omitted.

\section{Conclusions}

Generalized fuzzy entropy-based method segments an image using the threshold with membership value $m(m=0.1,0.2 \ldots 0.9)$. This method increases the opportunity of choosing appropriate thresholds, thus it 
obtains better performance than the fuzzy entropybased method. However, the results of generalized fuzzy entropy are not always satisfactory. Take Rice image for example (see Fig. 1), there are some object pixels having been wrong classified into background. This means that the step 0.1 for $m$ might be too coarse. However, too small step for $m$ would cause larger computation and increase the difficulty to select the result with the best visual effect. Therefore, how to automatically determine the parameter $m$ and evaluate the segmentation results are problems worth to research further.

\section{Acknowledgement}

This work is partially supported by National Nature Science Foundation of China (Grant No. 60572133)

\section{References}

[1] L.K. Huang and M.J. Wang, Image thresholding by minimizing the measures of fuzziness, Pattern Recognition, 28: 41-51, 1995.

[2] X.Q. Li, Z.W. Zhao and H.D. Cheng, Fuzzy entropy threshold approach to cancer detection, Information Sciences, 4: 49-56, 1995.

[3] H.D. Cheng, Y.H. Chen and Y, Sun, A novel fuzzy entropy approach to image enhancement and thresholding, Signal Processing, 75: 277301, 1999.

[4] A. De Luca and S. Termini, Definition of a nonprobabilistic entropy in the setting of fuzzy set theory, Information and control, 20: 301-312, 1972.

[5] L.A. Zadeh, Fuzzy sets, Information and Control, 8: 338-353, 1965.

[6] S.K. Pal, A note on the quantitative measure of image enhancement through fuzziness, IEEE Transactions on Pattern Analysis and Machine Intelligence, 4: 204-208, 1982.

[7] S.D. Zenzo, L. Cinque and S. Levialdi, Image thresholding using fuzzy entropies, IEEE Transactions on Systems, Man and Cybernetics. Part B, 28: 15-23, 1998.

[8] M. Sugeno, Fuzzy measures and fuzzy integrals: a survey, In: Gupta M, Saridis GN, Gaines BR (eds) Fuzzy Automata and Decision Processes, North Holland, Amsterdam and New York, pp. 89-102, 1977.

[9] R. Lowen, On fuzzy complements, Information Sciences, 14 : 107-113, 1978.

[10] R.R. Yager, On the measures of fuzziness and negation, Part II: Lattices, Information and Control, 44: 236-260, 1980.

[11] G. Malinowski, Many-Valued Logics. Oxford University Press, New York, 1993.
[12] H. Li, H.S. Yang, Fast and reliable image enhancement using fuzzy relaxation technique, IEEE Transactions on Systems, Man and Cybernetics, $19: 1276-1281,1989$.

[13] H.D. Cheng and J.R. Chen, Automatically determine the membership function based on the maximum entropy principle, Information Sciences, 96: 163-182, 1997. 